\title{
Correction to: \\ Effects of a Population Health Community-Based Palliative Care Program on Cost and Utilization by $L$ Yosick et al. (DOI: 10.1089/jpm.2018.0489)
}

\begin{abstract}
Fter publication of the article entitled, "Effects of a Population Health Community-Based Palliative Care Program on A Cost and Utilization" by L Yosick et al, (J Palliat Med 2019;22:1075-1081; DOI: 10.1089/jpm.2018.0489) an inadvertent error in the Institutional Review Board information was identified, and has been corrected in the version of record. The complete Institutional Review Board information is reproduced below, with the corrected information identified in bold:
\end{abstract}

\section{Author Disclosure Statement}

No competing financial interests exist.

This project was conducted as a Quality Improvement Initiative, and as such was not formally supervised by an Institutional Review Board.

The online version of this article has been corrected.

The authors regret this error. 